\title{
Transmission Eigenvalues in One Dimension
}

\author{
John Sylvester *
}

\begin{abstract}
We show how to locate all the transmission eigenvalues for a one dimensional constant index of refraction on an interval.
\end{abstract}

\section{Introduction}

The scattering operator for the time harmonic Helmholtz equation with compactly supported index of refraction $n(x)$ maps the asymptotics of incident waves (Herglotz wave functions) $v_{0}$ to the asymptotics of (outgoing) scattered waves $u^{+}$. Both $u^{+}$and $v^{0}$ are defined in $\mathbb{R}^{n}$ and satisfy

$$
\begin{gathered}
\left(\Delta+k^{2} n^{2}\right) u^{+}=\left(1-n^{2}\right) k^{2} v_{0} \\
\left(\Delta+k^{2}\right) v_{0}=0
\end{gathered}
$$

in all of $\mathbb{R}^{n}$.

If the scattering operator has a zero eigenvalue, we say that $k^{2}$ is a transmission eigenvalue [6], 4]. It follows from Rellich's theorem and unique continuation that the scattered wave $u^{+}$is identically zero outside any domain $D$ that contains the support of $n$, and therefore that $u=u^{+}$and $v=v^{0}$ are a pair of nontrivial functions satisfying

$$
\begin{array}{cc}
\left(\Delta+k^{2} n^{2}\right) u=\left(1-n^{2}\right) k^{2} v & \text { in } D \\
\left(\Delta+k^{2}\right) v=0 & \text { in } D \\
\left.u\right|_{\partial D}=\left.0 \quad \frac{\partial u}{\partial \nu}\right|_{\partial D}=0 &
\end{array}
$$

*Department of Mathematics,University of Washington, Seattle, Washington 98195,USA. (sylvest@uw.edu). Research was supported in part by NSF grant DMS1007447 
Whenever such a nontrivial pair exists, we say that $k^{2}$ is an interior transmission eigenvalue for the pair $(D, n)$ *

Remark 1. If we scale $v$ by introducing $w=k^{2} v$, then the $k^{2}$ on the right hand side of equation (1.1) disappears, and we see that the interior transmission eigenvalue problem is a true generalized eigenvalue problem which can be written as

$$
\begin{aligned}
\Delta u-\left(1-n^{2}\right) w & =-k^{2} n^{2} u \\
\Delta w & =-k^{2} w
\end{aligned}
$$

with the same boundary conditions as in (1.3) [9].

The analogous interior problem for obstacle scattering is the Dirichlet problem,

$$
\begin{gathered}
\left(\Delta+k^{2}\right) v=0 \quad \text { in } D \\
\left.v\right|_{\partial D}=0
\end{gathered}
$$

and the Dirichlet eigenvalues are fairly well understood. In particular, the Dirichlet eigenvalues of a one dimensional interval of length $L$ are exactly $\frac{n^{2} \pi^{2}}{L^{2}}$. The one dimensional interior transmission eigenvalue problem, even for a constant index of refraction, is not as simple. In one dimension, with the index of refraction $n(x)$ equal to the positive constant $\sigma$, equations (1.1) (1.2) (1.3) become

$$
\begin{gathered}
u^{\prime \prime}+k^{2} \sigma^{2} u=k^{2}\left(1-\sigma^{2}\right) v \\
v^{\prime \prime}+k^{2} v=0 \\
u\left( \pm \frac{L}{2}\right)=0 \quad u^{\prime}\left( \pm \frac{L}{2}\right)=0
\end{gathered}
$$

so that $k^{2}$ is an interior transmission eigenvalue if and only there exist a nontrivial pair of eigenfunctions $(u, v)$ satisfying (1.4) (1.5) (1.6).

We will show in proposition 4 that $k^{2}$ is an interior transmission eigenvalue if and only if $k$ is a root of the equation

$$
(\sigma-1)^{2} \sin ^{2}\left((\sigma+1) \frac{k l}{2}\right)-(\sigma+1)^{2} \sin ^{2}\left((\sigma-1) \frac{k l}{2}\right)=0
$$

${ }^{*}$ See section 8 of [3], as well as [8], for more details about the connection between transmission eigenvalues and interior transmission eigenvalues 
and the two theorems below describe the roots of (1.7), which we will call the interior transmission wavenumbers (positive square roots of interior transmission eigenvalues).

Theorem 2. The interior transmission wavenumbers satisfy

$$
|\operatorname{Im}(k)| \leq \frac{2}{L} \log \frac{3 \sigma+1}{|\sigma-1|}
$$

In theorem 3 below, the notation $\left\lfloor\frac{j}{m}\right\rfloor$ denotes the floor, the greatest integer less than or equal to $\frac{j}{m}$. The condition $\left\lfloor\frac{j}{m}\right\rfloor=\left\lfloor\frac{j+1}{m}\right\rfloor$ means that there is no integer between $\frac{j}{m}$ and $\frac{j+1}{m}$, and $\mathbb{Z}$ denotes the integers.

Theorem 3. Let $m=\frac{\sigma+1}{\sigma-1}$, and let $j \in \mathbb{Z}$

1. If $\frac{j}{m} \notin \mathbb{Z}$ and $\frac{j+1}{m} \notin \mathbb{Z}$, there are exactly two simple interior transmission wavenumbers in the strip

$$
\frac{2 j \pi}{(\sigma+1) L}<\operatorname{Re}(k)<\frac{2(j+1) \pi}{(\sigma+1) L}
$$

(a) If $\left\lfloor\frac{j}{m}\right\rfloor \neq\left\lfloor\frac{j+1}{m}\right\rfloor$, both are real

(b) If $\left\lfloor\frac{j}{m}\right\rfloor=\left\lfloor\frac{j+1}{m}\right\rfloor$ they are complex conjugates with nonzero imaginary parts

2. If $\frac{j}{m} \in \mathbb{Z}$, there is a quadruple interior transmission wavenumber at $k=\frac{j}{m}$ and no others in the strip

$$
\frac{2(j-1) \pi}{(\sigma+1) L}<\operatorname{Re}(k)<\frac{2(j+1) \pi}{(\sigma+1) L}
$$

The transmission wavenumbers for two $\sigma$ 's, with $L=2$, are plotted below. The vertical blue lines indicate the sets $\operatorname{Re}(k)=\frac{2 j \pi}{(\sigma+1) L}$. The roots that fall on these lines are quadruple.

TEs for $\sigma=1.25$

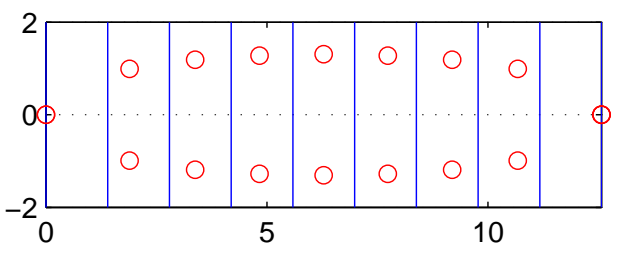

TEs for $\sigma=5$

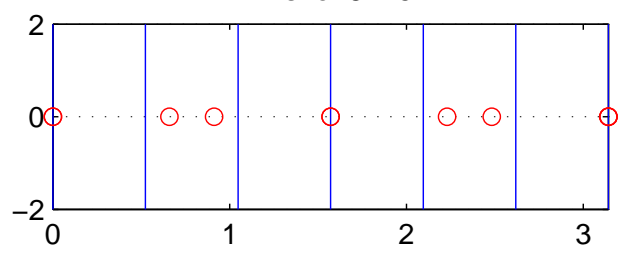

As these $\sigma$ 's are rational, the transmission eigenvalues are periodic. Remark 5 below explains how we did the computation. 


\section{Lemmas and Proofs}

Because we are dealing with constant coefficients, the transmission wavenumbers $k$ are the zeros of the analytic function on the left hand side of equation (1.7). This analytic function has two factors, and our proofs simplify if we treat each factor separately.

Proposition 4. $k$ is a transmission wavenumber if, and only if, $k$ satisfies

or

$$
(\sigma-1) \sin \left((\sigma+1) \frac{k l}{2}\right)=(\sigma+1) \sin \left((\sigma-1) \frac{k l}{2}\right)
$$

$$
(\sigma-1) \sin \left((\sigma+1) \frac{k l}{2}\right)=-(\sigma+1) \sin \left((\sigma-1) \frac{k l}{2}\right)
$$

The wavenumber $k$ satisfies (2.1) if there exists an eigenfunction pair $(u, v)$ of odd functions of $x$, and $k$ satisfies (2.2) if there exists an eigenfunction pair $(u, v)$ of even functions.

Proof. If a pair $u, v$ satisfies (1.4-1.6), then so do their odd and even parts, and the calculation is a little simpler for each of these parts. The odd parts satisfy (1.4,1.5) with (1.6) replaced by

$$
u(0)=v(0)=0 \quad u\left(\frac{L}{2}\right)=u^{\prime}\left(\frac{L}{2}\right)=0
$$

and the even parts satisfy (1.4 1.5) with (1.6) replaced by

$$
u^{\prime}(0)=v^{\prime}(0)=0 \quad u\left(\frac{L}{2}\right)=u^{\prime}\left(\frac{L}{2}\right)=0
$$

In the odd case, the boundary conditions at zero imply that

$$
v=\sin (k x)
$$

and

$$
u=-\sin (k x)+A \sin (k \sigma x)
$$

for some constant $A$. The boundary conditions at $x=\frac{L}{2}$ imply that

$$
\sin \left(\sigma k \frac{L}{2}\right) \cos \left(k \frac{L}{2}\right)=\sigma \sin \left(k \frac{L}{2}\right) \cos \left(\sigma k \frac{L}{2}\right)
$$


Noting that the left and right hand sides are the beats representation of the combination of the frequencies $(\sigma+1) \frac{L}{2}$ and $\left.(\sigma-1)\right) \frac{L}{2}$ suggests that we rewrite (2.4) as

$$
\left.(\sigma-1) \sin ((\sigma+1)) \frac{L}{2} k\right)=(\sigma+1) \sin \left((\sigma-1) \frac{L}{2} k\right)
$$

which is (2.1). A similar calculation shows the existence of even transmission eigenfunction pairs if and only if $k$ satisfies (2.2).

If we introduce the new variables

$$
m=\frac{\sigma+1}{\sigma-1}
$$

and

$$
z=(\sigma-1) k \frac{L}{2 \pi}
$$

then equations (2.1) and (2.2) become equations for a $z$ whcih depend on the single real parameter $m$. They become

$$
\begin{aligned}
& p_{m}^{o}(z):=\sin (m \pi z)-m \sin (\pi z)=0 \\
& p_{m}^{e}(z):=\sin (m \pi z)+m \sin (\pi z)=0
\end{aligned}
$$

where the superscripts refer to even and odd, respectively. Because $\sigma>0$, $|m|>1$. Because equations (2.6) and (2.7) remain the same if we change the sign of $m$, we may also assume that $m>1$, and will do so throughout

Remark 5. If $m=\frac{p}{q}$ is rational and we set $w=e^{\frac{i \pi z}{q}}$, then (2.6) becomes a polynomial equation in $w$.

$$
w^{2 p}-1=\frac{p}{q}\left(w^{p+q}-w^{p-q}\right)
$$

We can use a polynomial root finding algorithm to find the solutions and then take logarithms and rescale to find the interior transmission wavenumbers $k$. This is how we produced figure 1.

With the notation introduced in (2.5), theorem 2 becomes

\footnotetext{
$\dagger$ Alternatively, we could start with the assumption that $\sigma>1$, and then use the relationship between the transmission wavenumbers of $\sigma$ and those of $\frac{1}{\sigma}, k_{j}\left(\frac{1}{\sigma}\right)=\sigma k_{j}(\sigma)$ to show that our formulas and estimates apply to $\sigma<1$ as well.
} 
Theorem 6. If $z$ satisfies (2.6) or 2.7), then

$$
|\operatorname{Im}(z)| \leq \frac{\log (2 m+1)}{\pi(m-1)}
$$

Proof. If $z=x+i y$ satisfies (2.6)

$$
e^{m \pi|y|}-e^{-m \pi|y|} \leq 2|\sin m \pi z|=2 m|\sin \pi z| \leq m e^{\pi|y|}+m e^{-\pi|y|}
$$

so that

$$
e^{m \pi|y|} \leq e^{-m \pi|y|}+m e^{\pi|y|}+m e^{-\pi|y|}
$$

Dividing both sides by $e^{\pi|y|}$ gives

$$
e^{(m-1) \pi|y|} \leq e^{-(m+1) \pi|y|}+m+m e^{-2 \pi|y|} \leq 2 m+1
$$

Now taking the logarithm of both sides yields (2.9)

Locating the real parts of the roots of $p_{m}(z)=p_{m}^{o}(z) p_{m}^{e}(z)$ will take more work. We will show (roughly) that there are always two roots in certain of strips of the complex plane. We define

$$
\begin{aligned}
I_{\frac{j}{m}} & =\left\{x \mid \frac{j}{m}<x<\frac{j+1}{m}\right\} \\
S_{\frac{j}{m}} & =\left\{z \mid \frac{j}{m}<\operatorname{Re}(z)<\frac{j+1}{m}\right\}
\end{aligned}
$$

A restatement of theorem 3, using the notation of equation (2.5), is theorem 7 below.

Theorem 7. Let $m>1$.

1. If neither $\frac{j}{m}$ nor $\frac{j+1}{m}$ are integers, then $p_{m}$ has two simple roots in the strip $S_{\frac{j}{m}}$.

(a) If the interval $I_{\frac{j}{m}}$ contains an integer $k$, both roots are real, and one is bigger and the other smaller than $k$.

(b) If the interval $I_{\frac{j}{m}}$ does not contain an integer, the roots are complex conjugates (with nonzero imaginary parts).

2. If $\frac{j}{m}$ is an integer, $\frac{j}{m}$ is a quadruple root of $p_{m}$, and $p_{m}$ has no other roots in $S_{\frac{j}{m}}$ or $S_{\frac{j-1}{m}}$. 
Theorem 7 is an immediate consequence of the analogous theorems for $p_{m}^{o}$ and $p_{m}^{e}$. These theorems are a little more complicated to state, but easier to prove.

Theorem 8. Let $m>1$.

1. Suppose that neither $\frac{j}{m}$ nor $\frac{j+1}{m}$ are integers.

(a) If the interval $I_{\frac{j}{m}}$ contains an integer $k$ and $j+\left\lfloor\frac{j}{m}\right\rfloor$ is even, then $p_{m}^{o}$ has a real root in the interval $\left(\frac{j}{m}, k\right)$ and no other roots in $S_{\frac{j}{m}}$ and $p_{m}^{e}$ a root in $\left(k, \frac{j+1}{m}\right)$ and no other roots in $S_{\frac{j}{m}}$. If the interval $I_{\frac{j}{m}}$ contains an integer $k$ and $j+\left\lfloor\frac{j}{m}\right\rfloor$ is odd, then $p_{m}^{o}$ has a real root in the interval $\left(k, \frac{j+1}{m}\right)$ and no other roots in $S_{\frac{j}{m}}$ and $p_{m}^{e}$ a root in $\left(\frac{j}{m}, k\right)$ and no other roots in $S_{\frac{j}{m}}$.

(b) If the interval $I_{\frac{j}{m}}$ does not contain an integer and $j+\left\lfloor\frac{j}{m}\right\rfloor$ is odd, $p_{m}^{o}$ has 2 simple complex conjugate roots in $S_{\frac{j}{m}}$ and $p_{m}^{e}$ has none. If $j+\left\lfloor\frac{j}{m}\right\rfloor$ is even, $p_{m}^{e}$ has 2 simple complex conjugate roots in $S_{\frac{j}{m}}$ and $p_{m}^{o}$ has none.

2. If $\frac{j}{m}$ is an integer, neither $p_{m}^{o}$ nor $p_{m}^{e}$ have any roots in $S_{\frac{j}{m}}$ or $S_{\frac{j-1}{m}}$. If $j+\left\lfloor\frac{j}{m}\right\rfloor$ is even, $\frac{j}{m}$ is a triple root of $p_{m}^{o}$ and a simple root of $p_{m}^{e}$. If $j+\left\lfloor\frac{j}{m}\right\rfloor$ is odd, $\frac{j}{m}$ is a triple root of $p_{m}^{e}$ and a simple root of $p_{m}^{o}$.

We will prove theorem 8 (we will only prove the results for $p_{m}^{o}$ ) by studying a one parameter family of equations with $m$ fixed and parameter $\beta$.

$$
p_{\beta}(z)=\sin (m \pi z)-\beta \sin (\pi z)
$$

We will use the fact that that the roots of the entire function $p_{\beta}(z)$ depend continuously on the parameter $\beta^{\ddagger}$. We will also use the fact that, as $\beta \rightarrow \infty$, the roots of $p_{\beta}$ must approach those of $\sin (\pi z)$. This last fact follows from the first by multiplying (2.10) by $\alpha=\frac{1}{\beta}$, and the fact that the roots of $\alpha \sin (m \pi z)-\sin (\pi z)$ depend continuously on $\alpha$ as $\alpha$ approaches zero.

\footnotetext{
${ }^{\ddagger}$ The continuity of the roots of analytic functions follows from the fact that roots are isolated, together with the argument principle.
} 
Before giving the rigorous details, we give a rough outline of the proof of Theorem 8, To simplify this outline we ignore the case where $\frac{j}{m}$ is an integer.

Theorem 8 makes two assertions

1. It tells us the number of roots $p_{m}^{o}$ in each strip $S_{\frac{j}{m}}$. This number is the same for $p_{\beta}$ for all $\beta>0$.

2. It tells us the number of real roots in each strip when $\beta=m$. The number of real roots of $p_{\beta}$ changes as $\beta$ increases from zero to $m$, but is the same for all $\beta \geq m$.

We establish the number of roots in each strip by observing that:

1. at $\beta=0$ all roots are real and on the boundary of the strips $S_{\frac{j}{m}}$, and the derivatives are nonzero and pointing in the direction of the correct strip (lemma 9), so that, for small positive $\beta$, the correct number of roots have entered each strip(corollary 10).

2. Lemma 11 tells us that no roots can cross the boundary of $S_{\frac{j}{m}}$, so the correct number of roots remain in each strip for all $\beta$ (corollary 12).

We calculate the number of roots which are real at $\beta=m$ by observing that:

1. As $\beta$ approaches infinity, a single root must approach each integer (the roots of $\sin \pi z$ ), and the other roots must leave every compact subset of $\mathbb{C}$.

2. A calculation(lemma 13) shows that for $\beta \geq m$, all roots are simple.

3. This means that any real roots of $p_{\beta}$ in $S_{\frac{j}{m}}$ at $\beta=m$ must remain real for $\beta \geq m$ and remain in $S_{\frac{j}{m}}$, so they remain in the interval $I_{\frac{j}{m}}$. But, as $\beta$ approaches infinity, the roots of $p_{\beta}$ must either approach the roots of $\sin \pi z$ or leave every compact subset of $\mathbb{C}$. The roots in the $S_{\frac{j}{m}}$ 's that don't contain an integer must leave every compact set, so they cannot remain in the interval $I_{\frac{j}{m}}$, so they cannot have been real when $\beta=m$.

4. The $S_{\frac{j}{m}}$ 's that contain an integer contain only one root for all $\beta$, so the root remains simple and therefore real at $\beta=m$. That root approaches the integer as $\beta$ approaches infinity. 
The detailed proof follows:

Lemma 9. The roots of $p_{0}=\sin (m \pi z)$ are $\frac{j}{m}$. Each of these roots is simple, and thus continues to a unique root $z_{j}(\beta)$ of $p_{\beta}$ for small $\beta$. Moreover,

$$
\left.\frac{d z_{j}}{d \beta}\right|_{\beta=0}=(-1)^{j+\left\lfloor\frac{j}{m}\right\rfloor}\left|\sin \left(\frac{j \pi}{m}\right)\right|
$$

Proof. At $\beta=0$, the roots of $\sin m \pi z$ are located at $\frac{j}{m}$ and are all simple. The implicit function theorem implies that, for small $\beta$, they remain simple and depend analytically on $\beta$, so we can define $z_{j}(\beta)$ to be the continuation of that root. Differentiating (2.10) yields

so that

$$
\frac{d z_{j}}{d \beta}\left(m \pi \cos \left(m \pi z_{j}\right)+\beta \pi \cos \left(\pi z_{j}\right)\right)=\sin \left(\pi z_{j}\right)
$$

$$
\left.\frac{d z_{j}}{d \beta}\right|_{\beta=0}=\frac{\sin \frac{\pi j}{m}}{\cos \pi j}=(-1)^{j} \sin \frac{j \pi}{m}=(-1)^{j+\left\lfloor\frac{j}{m}\right\rfloor}\left|\sin \left(\frac{j \pi}{m}\right)\right|
$$

Knowledge of the derivatives tells us that the roots are in the correct strips for small $\beta$.

Corollary 10. For $\beta>0$ and sufficiently small, all roots of $p_{\beta}$ are real and:

1. Suppose that neither $\frac{j}{m}$ nor $\frac{j+1}{m}$ are integers.

(a) If the interval $I_{\frac{j}{m}}$ contains an integer $k$, then $p_{\beta}$ has a real root in the interval $\left(\frac{j}{m}, k\right)$ and no other roots in $S_{\frac{j}{m}}$ if $j+\left\lfloor\frac{j}{m}\right\rfloor$ is even. $p_{\beta}$ has a root in $\left(k, \frac{j+1}{m}\right)$ and no other roots in $S_{\frac{j}{m}}$ if $j+\left\lfloor\frac{j}{m}\right\rfloor$ is odd.

(b) If the interval $I_{\frac{j}{m}}$ does not contain an integer and $j+\left\lfloor\frac{j}{m}\right\rfloor$ is odd, $p_{\beta}$ has no roots in $S_{\frac{j}{m}}$. If $j+\left\lfloor\frac{j}{m}\right\rfloor$ is even, $p_{\beta}$ has two roots in $S_{\frac{j}{m}}$.

2. If $\frac{j}{m}$ is an integer, $\frac{j}{m}$ is a simple root of $p_{\beta}$. If $j+\left\lfloor\frac{j}{m}\right\rfloor$ is even, there is a simple root in each of $I_{\frac{j}{m}}$ and $I_{\frac{j-1}{m}}$. If $j+\left\lfloor\frac{j}{m}\right\rfloor$ is odd, there are no roots in $I_{\frac{j}{m}}$ or $I_{\frac{j-1}{m}}$. 
Proof. Because $\sin \bar{z}=\overline{\sin z}$, the roots of (2.6) must occur in conjugate pairs, and simple roots must remain real for small $\beta$. Lemma 9 tells us that, $z_{j}$ starts at $\frac{j}{m}$ and moves to the right if $\frac{j}{m}$ is not an integer and $j+\left\lfloor\frac{j}{m}\right\rfloor$ is even, or to the left if $\frac{j}{m}$ is not an integer and $j+\left\lfloor\frac{j}{m}\right\rfloor$ is odd. If $I_{\frac{j}{m}}$ does not contain an integer, then $\frac{j+1}{m}+\left\lfloor\frac{j+1}{m}\right\rfloor$ is odd whenever $j+\left\lfloor\frac{j}{m}\right\rfloor$ is even, and even whenever $j+\left\lfloor\frac{j}{m}\right\rfloor$ is odd, so that both $z_{j}$ and $z_{j+1}$ move into $I_{\frac{j}{m}}$ when $j+\left\lfloor\frac{j}{m}\right\rfloor$ is even, and away from $I_{\frac{j}{m}}$ when $j+\left\lfloor\frac{j}{m}\right\rfloor$ is odd, which establishes item $1 \mathrm{~b}$.

If $I_{\frac{j}{m}}$ contains an integer $k$, then $z_{j}$ moves into $I_{\frac{j}{m}}$ and $z_{j+1}$ moves away from $I_{\frac{j}{m}}$ when $j+\left\lfloor\frac{j}{m}\right\rfloor$ is even, and $z_{j_{+}}$moves into $I_{\frac{j}{m}}$ and $z_{j}$ moves away when $j+\left\lfloor\frac{j}{m}\right\rfloor$ is odd. The root which moves into $I_{\frac{j}{m}}$ remains in either $\left(\frac{j}{m}, k\right)$ or $\left(k, \frac{j+1}{m}\right)$ for small $\beta$, which establishes item 1a.

Finally, if $\frac{j}{m}$ is an integer, both terms in (2.10) vanish, so that $\frac{j}{m}$ remains a root for all $\beta$, and remains simple at least for $\beta$ small. If $\frac{j}{m}$ is an integer, $\frac{j-1}{m}+\left\lfloor\frac{j-1}{m}\right\rfloor=j+\left\lfloor\frac{j}{m}\right\rfloor-2$ and $\frac{j+1}{m}+\left\lfloor\frac{j+1}{m}\right\rfloor=j+\left\lfloor\frac{j}{m}\right\rfloor+1$, so $z_{j-1}$ moves into $I_{\frac{j-1}{m}}$ and $z_{j+1}$ moves into $I_{\frac{j+1}{m}}$ if $j+\left\lfloor\frac{j}{m}\right\rfloor$ is even, and both move away from those intervals if $j+\left\lfloor\frac{j}{m}\right\rfloor$ is odd. This establishes item 2 and finishes the proof.

At a root of $p_{\beta}(\mathrm{z})$, the real parts of the $\sin m \pi z$ must equal the real part of $\beta \sin \pi z$. The lemma below identifies the sets on which the real parts of these terms vanish.

Lemma 11. $\operatorname{Re}(\sin (z))=0$ iff $\sin (\operatorname{Re}(z))=0$

Proof.

$$
\sin (x+i y)=\sin x \cosh y+i \cos x \sinh y
$$

and $\cosh y$ never vanishes.

A consequence of lemma 11 is that $p_{\beta}$ has no $\operatorname{roots}$ on $\operatorname{Re}(z)=\frac{j}{m}$ or on $\operatorname{Re}(z)=k$, when $j$ or $k$ are integers, unless $\frac{j}{m}$ equals an integer. This is enough to show that the roots which entered each strip for small $\beta$, must remain there. 
Corollary 12. For all $0<\beta<\infty$

1. The conclusions in items 10 and 16 of corollary 10 , remain true.

2. If $\frac{j}{m}$ is an integer and $j+\left\lfloor\frac{j}{m}\right\rfloor$ is odd, $\frac{j}{m}$ is a simple root of $p_{\beta}$ and $p_{\beta}$ has no other roots in $S_{\frac{j}{m}}$ or $S_{\frac{j+1}{m}}$. If $\frac{j}{m}$ is an integer and $j+\left\lfloor\frac{j}{m}\right\rfloor$ is even, $p_{\beta}$ has three roots in $S_{\frac{j-1}{m}} \cup S_{\frac{j}{m}}$, one of which is $\frac{j}{m}$.

Proof. The hypothesis of item 1, that $\frac{j}{m}$ and $\frac{j+1}{m}$ are not integers, combine with lemma 11 to guarantee that $p_{\beta}$ can have no roots on the boundary of $S_{\frac{j}{m}}$. As the roots of the entire analytic function $p_{\beta}$ depend continuously on $\beta$, this implies that no root can enter or leave $S_{\frac{j}{m}}$. Thus, if $S_{\frac{j}{m}}$ contained no roots for small $\beta$, it contains no roots for all $\beta$. If $S_{\frac{j}{m}}$ contained two roots for some $\beta$, it contains two roots for all $\beta$. This establishes item $1 b$ for all $\beta$. To establish item 1a, note that lemma 11 forces the simple root to remain in $k<\operatorname{Re}(z)<\frac{j+1}{m}$ or $\frac{j}{m}<\operatorname{Re}(z)<k$. As the simple real root remains simple, it must also remain real.

The two statements in item 2 follow similarly from the fact that no root can cross $\frac{j+1}{m}$ or $\frac{j-1}{m}$, as these cannot be integers if $\frac{j}{m}$ is an integer because $m>1$.

Lemma 13. For $\beta>m$, all roots of $p_{\beta}$ are simple. For $\beta=m$, all roots are simple except $z=\frac{j}{m}$ in the case that $j+\frac{j}{m}$ is an even integer. In this case, $\frac{j}{m}$ is a triple root of $p_{\beta}$.

Proof. All roots of $p_{\beta}$ that aren't real are simple, because there are no more than three in any strip, and they must occur in conjugate pairs. Suppose we have a double real root, then

$$
\sin m \pi z=\beta \sin \pi z
$$

and

$$
m \cos m \pi z=\beta \cos \pi z
$$

Squaring both (2.12) and (2.13), and adding the results gives

$$
\sin ^{2} m \pi z+\cos ^{2} m \pi z+\left(m^{2}-1\right) \cos m \pi z=\beta^{2}
$$

or

$$
\cos ^{2} m \pi z=\frac{\beta^{2}-1}{m^{2}-1}
$$


which is impossible for $\beta>m$ because the right hand side is bigger than one. If $\beta=m$, then (2.14) and (2.13) are possible, but only if

$$
\cos \pi z=\cos m \pi z= \pm 1
$$

which implies that $z$ and $m z$ are either both even or both odd integers. This is equivalent to the statement that $z=\frac{j}{m}$ and $j+\left\lfloor\frac{j}{m}\right\rfloor$ is an even integer. One can check directly that the root is indeed triple in this case.

Proof of Theorem 8. We only prove the theorem for $p_{m}^{o}$, as the proof for $p_{m}^{e}$ is analogous. Item 1a follows immediately from item 1 of corollary 12. Item 1 of corollary 12 also implies all of item 10, except the fact that the two roots are not real. To see this, note that, as $\beta \rightarrow \infty$, the roots of $p_{\beta}$ must either approach the roots of $\sin \pi z$, which are integers, or leave every compact subset of the complex plane. Since $I_{\frac{j}{m}}$ contains no integers, the two roots in $S_{\frac{j}{m}}$ must leave every compact subset of $\mathbb{C}$. As $\operatorname{Re}(z)$ must remain between $\frac{j}{m}$ and $\frac{j+1}{m}$, the imaginary parts of these two roots must grow unbounded as $\beta \rightarrow \infty$. If they were real at $\beta=m$, lemma 13 would imply that they were simple and remained simple and real for all $\beta$, contradicting the fact that their imaginary parts grow unbounded as $\beta \rightarrow \infty$. Hence they must not have been real at $\beta=m$.

Item 2 follows for a similar reason. We can check directly that $\frac{j}{m}$ is either a simple or a triple root, depending on the parity of $j+\left\lfloor\frac{j}{m}\right\rfloor$. Item 2 of corollary 12 tells us that these are all of the roots in the closure of $S_{\frac{j-1}{m}} \cup S_{\frac{j}{m}}$, so there are no other roots of $p_{m}^{o}$ in these strips.

\section{Some Limiting Cases and Conclusions}

We have shown that there are two transmission wavenumbers in each closed strip $\frac{2 j \pi}{(\sigma+1) L} \leq \operatorname{Re}(k) \leq \frac{2(j+1) \pi}{(\sigma+1) L}$ of width $\frac{2 \pi}{(\sigma+1) L}$, and two real transmission wavenumbers in each of those strips which contains an integer. We show below that, in the limiting cases of a weak scatterer $(\sigma \rightarrow 1)$, there are no longer any real transmission wavenumbers, and in the limiting case of a strong scatterer $(\sigma \rightarrow \infty)$, all the transmission wavenumbers become real.

The Born, or linear approximation, is the limit as $\sigma \rightarrow 1$. We return to equation (1.7), set $\pi z=k L$, and let $\sigma \rightarrow 1$ to obtain 


$$
\pi z= \pm \sin \pi z
$$

Introducing the parameter $\beta$ in front of the $\pi z$ on the right hand side, and repeating the steps we used to prove theorem 7 , gives:

Theorem 14. For every integer $j$ except $j=-1$ and $j=0$, equations (3.1) have exactly two non-real complex conjugate roots in the strip $j \leq \operatorname{Re}(j) \leq$ $j+1$, and they lie in the interior of the strip. The only root in $-1 \leq \operatorname{Re}(z) \leq 1$ is the triple root at $z=0$.

The imaginary parts of these roots grow logarithmically. The precise asymptotics, which can be verified by substituting (3.2) into (3.1), are

$$
z_{j}^{ \pm}=\left(j+\frac{1}{2}\right) \pm i \log \left(2\left|j+\frac{1}{2}\right|\right)+O\left(\frac{\log \left(2\left|j+\frac{1}{2}\right|\right)}{\left(j+\frac{1}{2}\right)}\right)
$$

so that $k_{j}=\frac{\pi z_{j}}{L}$ satisfy

$$
k_{j}^{ \pm} \sim \frac{\pi}{L}\left(\left(j+\frac{1}{2}\right) \pm i \log \left(2\left|j+\frac{1}{2}\right|\right)\right)
$$

where, for the odd integers $j, z_{j}$ solve equation (3.1) with the plus sign, and, for even integers $j$, the $z_{j}$ solve (3.1) with the minus sign.

The limit as $\sigma \rightarrow \infty$ is a little more work to calculate. This is the limit as $m \rightarrow 1$, so that equation (2.7) becomes

$$
\sin \pi z=-\sin \pi z
$$

or

$$
\sin \pi z=0
$$

with roots at the integers. Thus, as $\sigma \rightarrow \infty$, the even transmission wavenumbers

$$
k_{j} \sim \frac{2 \pi j}{\sigma L}
$$

have the same asymptotics as the square roots of the eigenvalues of the Dirichlet problem

$$
\begin{gathered}
u^{\prime \prime}+k^{2} \sigma^{2} u=0 \\
u\left( \pm \frac{L}{2}\right)=0
\end{gathered}
$$


with odd $(u(x)=-u(-x))$ eigenfunctions.

The solutions of (2.6), which describe the transmission wavenumbers with odd eigenfunctions, have a different asymptotic behavior, that will match the asymptotics of a different self-adjoint boundary value problem. To see the limiting equation, we subtract $\sin \pi z$ from both sides of (2.6)

$$
\sin (m \pi z)-\sin \pi z=m \sin \pi z-\sin \pi z
$$

and divide by $m-1$

$$
\frac{\sin (m \pi z)-\sin \pi z}{m-1}=m \sin \pi z
$$

which becomes, as $m \rightarrow 1$

$$
\pi z \cos \pi z=\sin \pi z
$$

or

$$
\pi z=\tan \pi z
$$

The transmission wavenumbers satisfy

$$
k(\sigma-1) \frac{L}{2}=\tan k(\sigma-1) \frac{L}{2}
$$

and thus have the same asymptotics, as $\sigma \rightarrow \infty$, as the square roots of the eigenvalues of the self-adjoint boundary value problem.

$$
\begin{gathered}
v^{\prime \prime}+k^{2} \sigma^{2} v=0 \\
\pm \frac{L}{2} v^{\prime}\left( \pm \frac{L}{2}\right)=v\left( \pm \frac{L}{2}\right)
\end{gathered}
$$

with odd eigenfunctions $\$$.

Because these limiting wavenumbers are the square roots of the eigenvalues of a self-adjoint problem, we see that, as $\sigma \rightarrow \infty$, all transmission wavenumbers become real.

In our discussion of the one dimensional transmission eigenvalue problem, we have observed certain relationships between transmission eigenvalues,

\footnotetext{
§The wavenumbers of (3.6) with even eigenfunctions satisfy equation (3.5) with tangent replaced by cotangent
} 
Dirichlet eigenvalues, and the eigenvalues of another self-adjoint boundary value problem. We expect (optimistically) that some of these relationships will persist in higher dimensions. We know that, in the Born approximation, there are no real transmission eigenvalues [8], that a generic radially symmetric index of refraction has complex transmission eigenvalues [7], and that there are infinitely many transmission eigenvalues [8] [1]. All of these results are consistent with our one dimensional conclusions.

A consequence of theorem 3 is that the counting function for the number of interior transmission wavenumbers in a strip of width $W$ is

$$
N_{\mathbb{C}}(W ; \sigma, L)=\frac{(\sigma+1) L W}{\pi} \pm 2
$$

which has the same asymptotics as the counting function for Dirichlet wavenumbers for an interval of the same length with index of refraction $n=\sigma+1$.

The counting function for real interior transmission wavenumbers in an interval of width $W$ is

$$
N_{\mathbb{R}}(W ; \sigma, L)=\frac{(\sigma-1) L W}{\pi} \pm 2
$$

which matches the asymptotics of the counting function for Dirichlet wavenumbers for an interval of the same length with index of refraction $n=\sigma-1$ 田 It seems reasonable to conjecture that the relationship between the counting functions for the Dirichlet wavenumbers and the transmission wavenumbers remains true for non-constant $\sigma$, and in higher dimensions as well.

\section{References}

[1] Fioralba Cakoni, Drossos Gintides, and Houssem Haddar. The existence of an infinite discrete set of transmission eigenvalues. SIAM J. Math. Anal., 42(1):237-255, 2010.

[2] David Colton, Andreas Kirsch, and Lassi Päivärinta. Far-field patterns for acoustic waves in an inhomogeneous medium. SIAM J. Math. Anal., 20(6):1472-1483, 1989.

\footnotetext{
T This is correct as is for irrational $\sigma$. For rational $\sigma$ we need to adopt the convention that every quadruple root (there will always be some) is counted as two real roots and two non-real roots.
} 
[3] David Colton and Rainer Kress. Inverse acoustic and electromagnetic scattering theory, volume 93 of Applied Mathematical Sciences. Springer, New York, third edition, 2013.

[4] David Colton and Peter Monk. The inverse scattering problem for timeharmonic acoustic waves in an inhomogeneous medium. Quart. J. Mech. Appl. Math., 41(1):97-125, 1988.

[5] David Colton, Lassi Päivärinta, and John Sylvester. The interior transmission problem. Inverse Probl. Imaging, 1(1):13-28, 2007.

[6] Andreas Kirsch. The denseness of the far field patterns for the transmission problem. IMA J. Appl. Math., 37(3):213-225, 1986.

[7] Yuk-J Leung and David Colton. Complex transmission eigenvalues for spherically stratified media. Inverse Problems, 28(7):075005, 9, 2012.

[8] Lassi Päivärinta and John Sylvester. Transmission eigenvalues. SIAM J. Math. Anal., 40(2):738-753, 2008.

[9] John Sylvester. Discreteness of transmission eigenvalues via upper triangular compact operators. SIAM J. Math. Anal., 44(1):341-354, 2012. 